\title{
A compact streak camera for 150 fs time resolved measurement of bright pulses in ultrafast electron diffraction
}

\author{
G. H. Kassier, ${ }^{1}$ K. Haupt, ${ }^{1}$ N. Erasmus, ${ }^{1}$ E. G. Rohwer,${ }^{1}$ H. M. von Bergmann, ${ }^{1}$ \\ H. Schwoerer, ${ }^{1}$ S. M. M. Coelho, ${ }^{2}$ and F. D. Auret ${ }^{2}$ \\ ${ }^{1}$ Laser Research Institute, University of Stellenbosch, Private Bag X1, 7602 Matieland, South Africa \\ ${ }^{2}$ Department of Physics, University of Pretoria, Pretoria 0002, South Africa
}

(Received 25 June 2010; accepted 24 August 2010; published online 14 October 2010)

\begin{abstract}
We have developed a compact streak camera suitable for measuring the duration of highly charged subrelativistic femtosecond electron bunches with an energy bandwidth in the order of $0.1 \%$, as frequently used in ultrafast electron diffraction (UED) experiments for the investigation of ultrafast structural dynamics. The device operates in accumulation mode with 50 fs shot-to-shot timing jitter, and at a $30 \mathrm{keV}$ electron energy, the full width at half maximum temporal resolution is $150 \mathrm{fs}$. Measured durations of pulses from our UED gun agree well with the predictions from the detailed charged particle trajectory simulations. (C) 2010 American Institute of Physics.

[doi:10.1063/1.3489118]
\end{abstract}

\section{INTRODUCTION}

Ultrafast electron diffraction (UED) is a rapidly emerging technique that has been used to study structural dynamics of matter with sub-Angstrom spatial and subpicosecond temporal resolution. ${ }^{1-3}$ This is achieved by employing standard femtosecond laser technology in an optical-pump, high energy electron (10-100 keV range) probe setup. In studying samples that cannot be repeatedly excited and probed, it has been necessary to develop high brightness compact UED sources that can deliver subpicosecond electron pulses containing about $10^{4}$ electrons per pulse. ${ }^{1}$ An even higher brightness up to the $10^{6} e^{-}$/pulse range is envisioned for future sources. ${ }^{4,5}$ Space charge forces in highly charged bunches containing about $10^{4} e^{-} /$pulse lead to a relatively large energy bandwidth in the order of $0.1 \%$, which for the most commonly used table-top subrelativistic UED sources implies rapid temporal pulse broadening. ${ }^{6}$ If accelerated to $30 \mathrm{keV}$ electron energy, such pulses debunch at a rate of about 100 fs/cm propagation distance, and therefore, their temporal characterization with $\sim 100$ fs resolution would require compact streak cameras with a millimeter scale streak plate length to avoid temporal smearing. Currently, streak cameras employed in UED have streak plate lengths in the few centimeter range, and they also suffer from poor sensitivity (small streak velocity). This limits the attainable temporal resolution of such streak cameras to not much better than 1 $\mathrm{ps},{ }^{1,2,7}$ and the current state of the art table-top UED temporal characterization featuring $\sim 300$ fs resolution is in fact achieved by a laser pulse electron pulse cross correlation technique. ${ }^{8}$ While higher sensitivity can be attained with traveling wave deflectors, ${ }^{9}$ such streak plate geometries would be rather difficult to fabricate on the small length scales required for UED.

In this article, we present our realization of a streak camera with a millimeter scale streak plate length $L_{p}$ and submillimeter plate separation. The compact nature of the device not only minimizes temporal smear due to the finite electron energy bandwidth, but also allows a larger electric field between the streak plates than what is possible with centimeterscale streak cameras, thereby significantly enhancing the sensitivity. ${ }^{10}$ The compact design and consequent large operating frequency are also conducive toward low (sub-100 fs) shot-to-shot photoswitch timing jitter, thereby allowing the device to operate in accumulation mode. ${ }^{9}$

\section{EXPERIMENTAL AND RESULTS}

Figure 1(a) depicts the streak camera setup. The electron pulses to be characterized are produced by a custom-built compact dc photogun containing a back-illuminated thin film gold photocathode that is triggered by frequency tripled femtosecond laser pulses $(\lambda=258 \mathrm{~nm})$ from a commercial chirped pulse amplifier (Clark MXR CPA 2101). The photogenerated electron pulses are accelerated to $30 \mathrm{keV}$ energy through a $4 \mathrm{~mm}$ gap, and are then collimated by a compact solenoid lens. The streak camera itself was placed at a distance of $5 \mathrm{~cm}$ from the photocathode. As shown in Fig. 1(c), it consists of a pair of $3 \mathrm{~mm} \times 3 \mathrm{~mm}$ streak plates with a plate separation of $0.5 \mathrm{~mm}$ that are shunted through a low inductance $(\sim 5 \mathrm{nH})$ discharge loop containing a photoswitch that is placed as close as possible to the streak plates. The capacitor formed by the streak plates and parasitic capacitance of the photoswitch $(\sim 0.2 \mathrm{pF})$ is charged by an external pulsed voltage supply via a pair of decoupling resistors of value $R_{d}$. The operation is as follows: a $50 \mathrm{~ns}$ high voltage pulser that is synchronized to the amplified femtosecond laser charges the streak plates to a potential difference of $1.5 \mathrm{kV}$. A pulsed voltage source is required to reduce the switch duty cycle since thermal effects in the GaAs wafer cause switch instability and breakdown at a few hundred volts if a dc source is used. In addition, very short charging pulses mitigate the danger of arcing due to vacuum breakdown at the GaAs surface. Once fully charged, the trigger laser pulse impinges on the photoswitch, thereby initiating a damped harmonic oscillation of the effective LCR circuit 


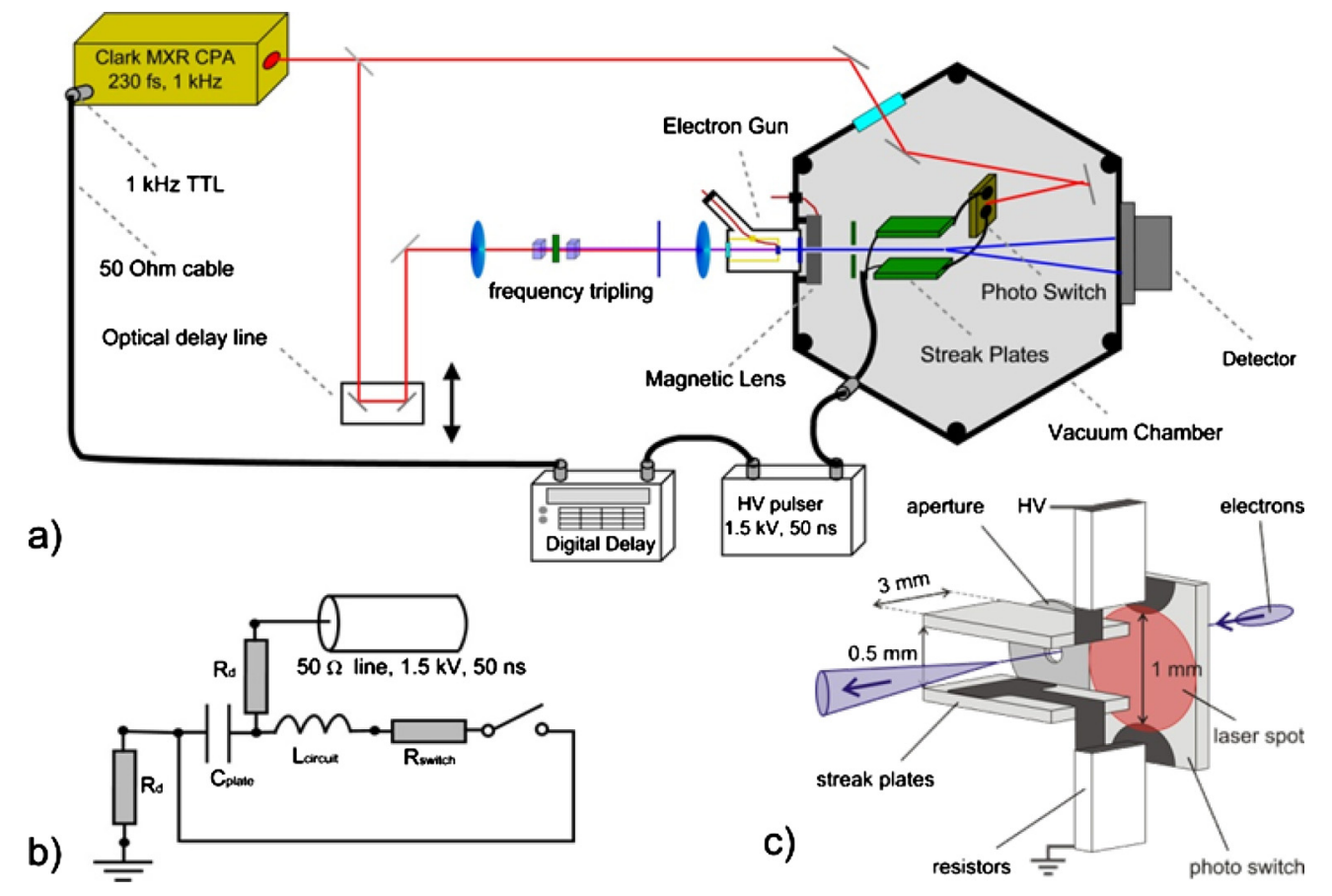

FIG. 1. (Color online) Compact streak camera setup and design. (a) Overview of the experimental setup, (b) lumped circuit diagram of the streak camera, and (c) schematic drawing of the streak camera design (not to scale).

[Fig. 1(b)]. By measuring the beam deflection as a function of photoswitch trigger delay time, it was possible to determine the temporal evolution of the streak electric field, as shown in Fig. 2. By fitting the transient to a simple damped oscillator model, a frequency of $5.2 \mathrm{GHz}$ and a circuit quality factor of $Q=3$ were obtained. In the streaking experiment, the electron pulse transits the streak field symmetrically in time about its first zero crossing, utilizing the maximum electric field ramp while ensuring zero net beam deflection. Shaping of the electron pulse by a $50 \mu \mathrm{m}$ aperture prior to injection into the streak field minimizes distortions of the streak image due to beam size-related factors such as streak plate misalignment and fringe fields. It also suppresses space charge-induced radial broadening of the pulses in the post-

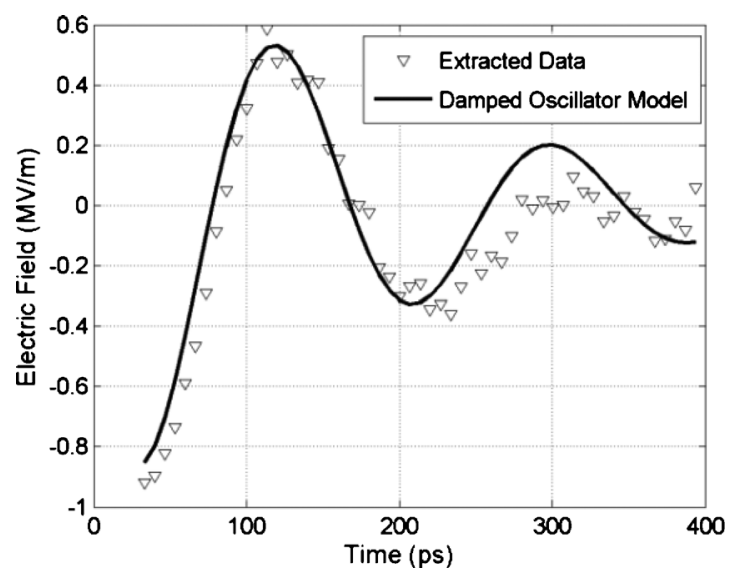

FIG. 2. Streaking electric field vs time. The electric field values (triangles) were calculated from recorded electron pulse deflection angles for different photoswitch trigger delay times. The data are well fitted by a simple damped oscillator model with a frequency of $5.2 \mathrm{GHz}$ and circuit quality factor $Q$ $=3$. This measurement was performed using a peak charging voltage of 400 $\mathrm{V}$. streak plate drift space. ${ }^{11}$ The photoswitch itself was made as follows: two $1.5 \mathrm{~mm}$ diameter circular Ohmic contacts with a minimum edge separation of $1 \mathrm{~mm}$ were fabricated by evaporating titanium $(5 \mathrm{~nm}) /$ germanium-gold $(200 \mathrm{~nm}) /$ gold $(200 \mathrm{~nm})$ onto a $0.5 \mathrm{~mm}$ thick semi-insulating gallium arsenide (GaAs) wafer (dark resistivity of $\sim 10^{6} \Omega \mathrm{cm}$ ) via electron beam deposition and subsequent annealing at $450{ }^{\circ} \mathrm{C}$ for $2 \mathrm{~min}$ in an argon filled furnace. The function of the decoupling resistors $R_{d}$ is to prevent interference of the charging circuit with the streak plate oscillations. The recharging time must however be significantly smaller than the time delay between the laser prepulse and the main pulse (about 4 ns in the present case) in order to suppress the destabilizing effect of the laser prepulse. ${ }^{9,12,13}$ Choosing $R_{d}$ $=2 \mathrm{k} \Omega$ yields a recharging time constant of about $1 \mathrm{~ns}$ (roughly equal to the measured carrier lifetime of the photoswitch) while ensuring sufficient decoupling during the high frequency ringing.

We now discuss the temporal resolution capability of the streak camera. The rms impulse response $\sigma_{\text {imp }}$ of the streak camera is given as the ratio of the rms beam size and the streak velocity at the detector. Due to the negligible aperture size and large camera length (about $35 \mathrm{~cm}$ in our case), the former is given in terms of the camera length $l_{c}$ and local rms angular spread of the beam $\sigma_{\theta}$ by $l_{c} \times \sigma_{\theta}$. The streak velocity is given by $v_{s}=l_{c} \times \omega_{s}$ where $\omega_{s}$ is the angular streak velocity. The impulse response can thus be written as

$$
\sigma_{\text {imp }}=\frac{\sigma_{\theta}}{\omega_{s}},
$$

which in turn is in the same order of magnitude as the temporal resolution associated with the data extraction which we denote as $\Delta \tau_{e}$. With the current electron gun and streak camera operating parameters, $\omega_{s}$ was measured to be $5.2 \mathrm{mrad} / \mathrm{ps}$ 


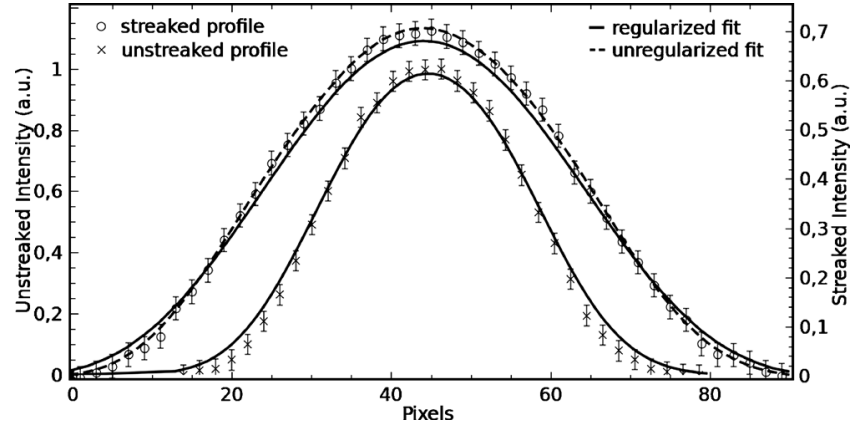

FIG. 3. Top: measured streak profile with error bars and fits corresponding to no regularization (dashed line) and optimal regularization (solid line), respectively. The best fit is obtained for no regularization $\left(\chi^{2}=0.1\right)$ while the regularized fit yields $\chi^{2}=2$. Bottom: unstreaked profile with fit corresponding to optimal regularization.

(Ref. 14) by recording the electron beam position on the detector as a function of the delay time between the electron pulse and the laser trigger pulse. By measuring the rms radius of the unstreaked spot on the detector, $\sigma_{\theta}$ was found to be $0.5 \mathrm{mrad}$, yielding an impulse response in the order of 100 fs according to Eq. (1).

The temporal resolution of the streak camera is determined by the temporal resolution due to data extraction $\Delta \tau_{e}$ as well as limitations on the temporal resolution due to the shot-to-shot timing jitter $\Delta \tau_{j}$ and the possibility of streak image broadening $\Delta \tau_{m}$ due to shear-type misalignment of the streak plates. We first discuss the temporal resolution due to data extraction $\Delta \tau_{e}$, which depends not only on the impulse response, but also on the fidelity of the data (noise level) in conjunction with the data extraction method. In order to extract the temporal electron density profile of the pulse, both the streaked and unstreaked images were binned along the axis perpendicular to the streak direction. Thus, the generated streaked profile can be interpreted as a convolution of the unstreaked profile with the temporal electron density profile (see insets in Fig. 3 for the recorded streaked and unstreaked images). Mathematically, this statement can be expressed as

$$
\mathbf{s}=\mathrm{Ad} ; \mathrm{A}=\left[\mathbf{u}_{1}, \mathbf{u}_{2}, \ldots, \mathbf{u}_{\mathbf{n}}\right],
$$

where $\mathbf{s}$ and $\mathbf{d}$ are column vectors representing the measured streaked profile and the determined electron density streak profile (which is, of course, linearly related to the temporal electron density profile via the streak velocity), respectively. The convolution matrix A consists of column vectors $\mathbf{u}_{\mathbf{i}}$ that contain the unstreaked profile data at various shifted index positions for each $\mathbf{u}_{\mathbf{i}}$ (the remaining entries of $\mathbf{u}_{\mathbf{i}}$ are set to zero). Inversion of the convolution operator A to extract the electron density profile is an ill-posed problem, and therefore one cannot simply solve for $\mathbf{d}$ by minimizing $\|$ Ad $-\mathbf{s} \|^{2}$. Instead, we solve the following optimization problem with non-negativity constraints on $\mathbf{d}$ :

$$
\min _{\mathbf{d} \geq 0}\left\{\frac{1}{2} \| \text { Ad }-\mathbf{s}\left\|^{2}+\frac{\gamma}{2}\right\| \mathbf{d} \|^{2}\right\} .
$$

Inclusion of the term involving the regularization parameter $\gamma>0$ in Eq. (3) is known as Tikhonov regularization. ${ }^{15}$ Assuming a fixed Gaussian variance $\delta^{2}$ for each measured data

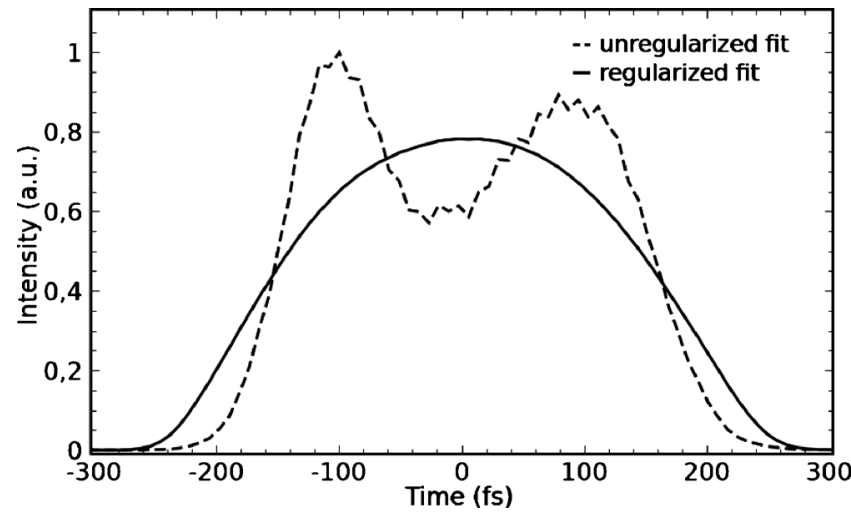

FIG. 4. Extracted temporal electron density profiles corresponding to no regularization (dashed line) and optimal regularization (solid line), respectively. The unregularized profile exhibits a pronounced double-humped feature which is likely an artifact and absent in the regularized profile.

point in $\mathbf{u}$ and $\mathbf{s}$, the reduced chi-squared statistic is approximated by

$$
\chi^{2} \approx \frac{1}{N \delta^{2}}\|\mathrm{~A} \mathbf{d}-\mathbf{s}\|^{2}
$$

for large $N=\operatorname{size}(\mathbf{s})$. Choosing $\gamma$ such that $\|\mathrm{Ad}-\mathbf{s}\|^{2}=N \delta^{2}$ yields $\chi^{2}=1$, i.e., with optimal regularization, we enforce a mean square error of the data fit which matches the expected uncertainty in the data. To solve for $\mathbf{d}$, Eq. (3) can be written as a quadratic programming problem in standard form, ${ }^{16}$

$$
\min _{\mathbf{d} \geq 0}\left\{\frac{1}{2} \mathbf{d}^{\mathrm{t}}\left(\mathrm{A}^{\mathrm{t}} \mathrm{A}+\gamma \mathrm{I}\right) \mathbf{d}-\mathbf{d}^{\mathrm{t}} \mathrm{A}^{\mathrm{t}} \mathbf{s}\right\}
$$

Here, $\mathrm{I}$ is the identity matrix, and the superscripted $\mathrm{t}$ denotes the transpose of the relevant vector or matrix. To estimate the temporal resolution associated with the data extraction $\Delta \tau_{e}$, consider the measured streaked and unstreaked profiles obtained from pulses containing 1500 electrons as plotted in Fig. 3. The error bars on the data were estimated by statistically analyzing a set of profiles recorded under the same conditions. The streaked profile was deconvolved subject to two regularization conditions: (1) $\gamma=0$ (no regularization) and (2) $\gamma>0$ subject to $\|\mathrm{Ad}-\mathbf{s}\|^{2}=N \delta^{2}$ ( $\delta$ is the rms error). For condition (1), one obtains the best fit (best correspondence of $\mathbf{s}$ and the reconstructed profile Ad, both plotted in Fig. 3) with $\chi^{2}=0.1$, and the corresponding full width at half maximum (FWHM) temporal duration is $\tau_{\mathrm{opt}}=240 \mathrm{fs}$ (see Fig. 4). The extracted pulse width increases to $256 \mathrm{fs}$ with proper regularization according to condition (2), in which case $\chi^{2}=1.1$. We interpret the pulse duration extracted with proper regularization to be the maximum pulse duration $\tau_{\max }$ consistent with the measured data (both the extracted pulse duration and $\chi^{2}$ increase monotonously with $\gamma$ so that stronger regularization would no longer lead to a data fit within the determined error bounds). As seen in Fig. 4, proper regularization eliminates the somewhat artificial double humped feature in the profile extracted without regularization. To determine the minimum pulse duration consistent with the measured data, consider the unstreaked profile shown in Fig. 3. Deconvolution of $\mathbf{u}$ itself should ideally yield zero pulse duration as $\mathbf{u}$ is indistinguishable from a streaked profile that one would have measured of an infi- 
nitely short pulse. Yet the width extracted with proper regularization $\left(\chi^{2}=1.1\right.$, the fit is plotted on top of the unstreaked profile in Fig. 3) is equal to $\tau_{0}=85$ fs FWHM. We therefore argue that the minimum pulse duration due to data extraction $\tau_{\text {min, } \mathrm{e}}$ should be given by $\left(\tau_{\text {min,e }}\right)^{2}=\left(\tau_{\text {opt }}\right)^{2}-\left(\tau_{0}\right)^{2}=(240 \mathrm{fs})^{2}$ $-(85 \mathrm{fs})^{2}$, yielding $\tau_{\min , \mathrm{e}}=225 \mathrm{fs}$. Another limitation to the temporal resolution is the shot-to-shot timing jitter of the streak camera caused by variation of the photoswitch resistance in response to trigger laser intensity fluctuations. ${ }^{9,12}$ To estimate the jitter, the center position of the streaked spot on the detector was measured as a function of trigger laser intensity yielding a sensitivity of $37 \mathrm{fs}$ per percentage intensity variation at the currently employed laser fluence of $1 \mathrm{~mJ} / \mathrm{cm}^{2} /$ pulse. With a measured relative FWHM laser intensity spread of $1.4 \%$, the estimated timing jitter is $\Delta \tau_{j}$ $=50$ fs. Lastly, assuming up to $10^{\circ}$ shear-type misalignment of the streak plates, an additional broadening of $\Delta \tau_{m}$ $=60 \mathrm{fs}$ can be expected due to the electron beam size of $50 \mu \mathrm{m}$. Since these effects exclusively act to broaden the measured pulse duration, they further reduce the minimum pulse duration consistent with the data extraction $\tau_{\text {min,e }}$ by $\tau_{\min }=\left[\left(\tau_{\text {min, },}\right)^{2}-\left(\Delta \tau_{j}\right)^{2}-\left(\Delta \tau_{\mathrm{m}}\right)^{2}\right]^{1 / 2}=210$ fs. The temporal resolution of the streak camera is then estimated as $\Delta \tau=\left[\left(\tau_{\max }\right)^{2}-\left(\tau_{\min }\right)^{2}\right]^{1 / 2}=\left[(256 \mathrm{fs})^{2}-(210 \mathrm{fs})^{2}\right]^{1 / 2}=150 \mathrm{fs}$. While the temporal duration of the pulse containing 1500 electrons could be written as $235 \pm 25$ fs, we prefer to state the value obtained with the properly regularized deconvolution $\tau_{\max }=256 \mathrm{fs}$ with the understanding that $\left[\left(\tau_{\max }\right)^{2}\right.$ $\left.-\left(\tau_{\min }\right)^{2}\right]^{1 / 2}=150 \mathrm{fs}$. This relation also holds for pulse durations other than $256 \mathrm{fs}$.

Using an autocorrelator, the pulse width of the laser was measured as $230 \pm 20$ fs FWHM. By simulating the evolution of the pulse duration through the various optics including nonlinear crystals and various dispersive media, the duration of the frequency tripled pulse at the photocathode was found to be $210 \pm 20$ fs. Taking into account the initial Gaussian radial profile of the laser pulse on the cathode with a FWHM radius of $190 \mu \mathrm{m}$, the measured beam radius of $270 \mu \mathrm{m}$ at the streak camera position $5 \mathrm{~cm}$ from the photocathode, and the fact that the beam is shaped by the $50 \mu \mathrm{m}$ aperture, the temporal electron density profile of the electron bunches could be accurately simulated using the ASTRA code. ${ }^{17}$ The simulated and measured profiles (regularization with $\chi^{2}=1.1$ ) for the cases of 1500 and 8000 electrons per pulse, respectively, are shown in Fig. 5. Excellent agreement between simulated and measured pulse durations is evident for both cases considered, with the simulated (measured) FWHM pulse durations for the 1500 and $8000 e^{-} /$pulse cases being $228 \mathrm{fs}$ ( $256 \mathrm{fs}$ ) and $350 \mathrm{fs}$ ( $345 \mathrm{fs}$ ), respectively. These results serve as further confirmation of the estimated $150 \mathrm{fs}$ temporal resolution. Interestingly enough, the doublehumped shape of the $\sim 250$ fs pulse that disappears with regularization (see Fig. 4) remains visible in case of the regularized $\sim 350$ fs profile in Fig. 5. This feature, which is statistically significant given the error bars of the measured streak profile, also shows up in the simulated profile given in Fig. 5. While the simulated $\sim 250$ fs profile also appears to exhibit a double-humped shape, this cannot be resolved by the current streak camera. The good qualitative agreement of

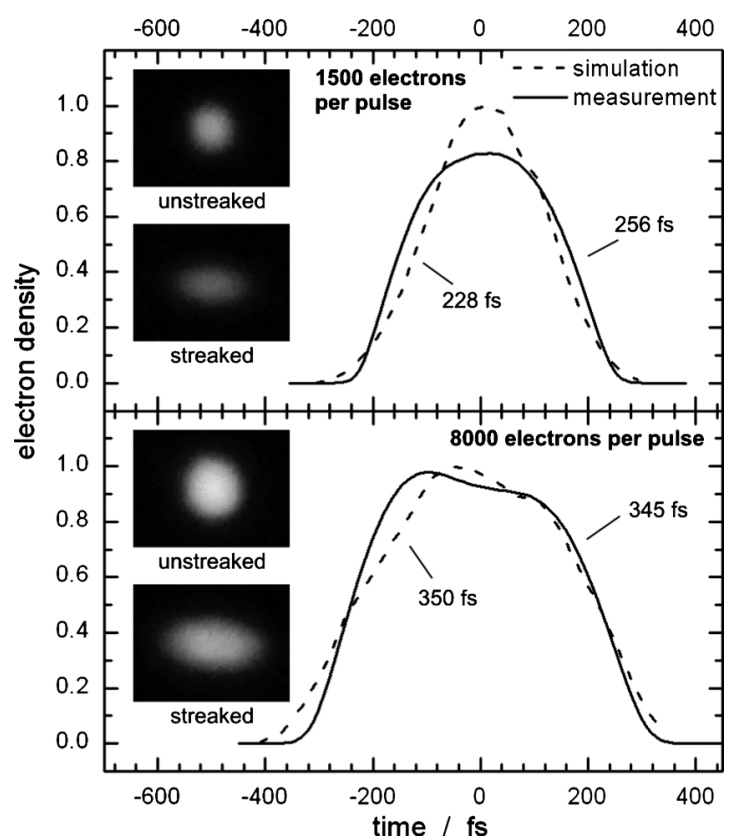

FIG. 5. Measured and simulated longitudinal electron density profiles of pulses containing 1500 electrons and 8000 electrons, respectively. The intensities of the simulated and measured profiles are normalized so as to correspond to the same total electron number. The insets depict the recorded streak images, and the FWHM pulse durations are also shown.

measurement and simulation, particularly in case of the $\sim 350$ fs pulse, confirms the ability of our device to measure not only the duration, but also the temporal electron density profile of ultrashort electron pulses.

\section{CONCLUSION}

In summary, a simple and compact streak camera with 150 fs temporal resolution for measurement of highly charged subrelativistic electron pulses has been constructed and thoroughly characterized. Good agreement of measured pulse durations and temporal profiles with simulations confirms the utility of the device for diagnostics of current state of the art UED guns delivering highly charged sub-300 fs electron pulses.

\section{ACKNOWLEDGMENTS}

We would like to thank Paul Papka for the use of his beam energy analyzer setup. This work is based upon research supported by the South African Research Chairs Initiative of the Department of Science and Technology and the National Research Foundation.

${ }^{1}$ B. J. Siwick and R. J. D. Miller, Chem. Phys. 299, 285 (2004).

${ }^{2}$ J. R. Dwyer, C. T. Hebeisen, R. Ernstorfer, M. Harb, V. B. Deyirmenjian, R. E. Jordan, and R. J. D. Miller, Philos. Trans. R. Soc. London, Ser. A 364, 741 (2006).

${ }^{3}$ H. Ihee, V. A. Lobastov, U. M. Gomez, B. M. Goodson, R. Srinivasan, C.-Y. Ruan, and A. H. Zewail, Science 291, 458 (2001).

${ }^{4}$ T. van Oudheusden, E. F. de Jong, S. van der Geer, W. P. E. M. Op 't Root, and O. J. Luiten, J. Appl. Phys. 102, 093501 (2007).

${ }^{5}$ G. H. Kassier, K. Haupt, N. Erasmus, E. G. Rohwer, and H. Schwoerer, J. Appl. Phys. 105, 113111 (2009).

${ }^{6}$ B. J. Siwick, J. R. Dwyer, R. E. Jordan, and R. J. D. Miller, J. Appl. Phys. 92, 1643 (2002).

${ }^{7}$ X. Wang, S. Nie, H. Park, J. Li, R. Clinite, R. Li, X. Wang, and J. Cao, 
Rev. Sci. Instrum. 80, 013902 (2009)

${ }^{8}$ C. T. Hebeisen, G. Sciaini, M. Harb, R. Ernstorfer, T. Dartigalongue, S. G. Kruglik, and R. J. D. Miller, Opt. Express 16, 3334 (2008).

${ }^{9}$ J. Liu, J. Wang, B. Shan, C. Wang, and Z. Chang, Appl. Phys. Lett. 82, 3553 (2003)

${ }^{10}$ The accelerated transverse motion of electrons leads to a net transverse displacement inside the streak region that scales as $L_{p}{ }^{2}$. Assuming that there is a constant ratio between plate separation and $L_{p}$, the available space for the transverse displacement scales linearly with $L_{p}$. It follows that the maximum electric field magnitude can be applied, and therefore, the maximum possible sensitivity is proportional to $L_{p}{ }^{-1}$.

${ }^{11}$ At a fixed charge density, the total charge $Q$ passing through the aperture of radius $r$ scales as $r^{2}$. Assuming for simplicity that the apertured pulse is a disc (specifically a flat spheroid), it follows that the electrostatic potential energy per electron scales as $Q / r$ (see Ref. 4). If all the potential energy is converted to transverse kinetic energy, it follows that the transverse momentum, and hence the transverse angular spread $\sigma_{\theta}$, is proportional to $r^{1 / 2}$. For sufficiently small $r$ and long pulse durations, the apertured pulse becomes cylindrical, resulting in a more rapid decrease of the angular spread with $r$. In any case, $\sigma_{\theta}$ decreases with decreasing aperture size.

${ }^{12}$ C. Belzile, J. C. Kieffer, C. Y. Cote, T. Oksenhendler, and D. Kaplan, Rev. Sci. Instrum. 73, 1617 (2002).

${ }^{13}$ C. Bonté, M. Harmand, F. Dorchies, S. Magnan, V. Pitre, J. C. Kieffer, P. Audebert, and J. P. Geindre, Rev. Sci. Instrum. 78, 043503 (2007).

${ }^{14}$ This corresponds to an electric field ramp rate of $10 \mathrm{kV} / \mathrm{mm} / \mathrm{ns}$.

${ }^{15}$ V. Y. Arsenin and A. N. Tikhonov, Solutions of Ill-Posed Problems (John Wiley \& Sons, New York, 1977).

${ }^{16}$ G. Landi and F. Zama, Appl. Math. Comput. 175, 715 (2006).

${ }^{17}$ See www.desy.de/ mpyflo/. 